\title{
Towards effective and stable probiotics
}

\author{
D.R. Yarullina, ${ }^{\mathrm{a}, *}$, L.G. Damshkaln ${ }^{\mathrm{b}}$, N.L. Bruslik ${ }^{\mathrm{a}}$, O.A. Konovalova ${ }^{\mathrm{c}}$, O.N. Ilinskaya ${ }^{\mathrm{a}}$ \\ and V.I. Lozinsky \\ ${ }^{a}$ Department of Microbiology, Kazan (Volga Region) Federal University, Kazan, Russia \\ ${ }^{\mathrm{b}}$ Institute of Element-Organic Compounds, Russian Academy of Sciences, Moscow, Russia \\ ${ }^{\mathrm{c} D e p a r t m e n t}$ of Optics and Nanophotonics, Kazan (Volga region) Federal University, Kazan, Russia \\ *Corresponding author: E-mail: kasfes@gmail.com
}

\begin{abstract}
BACKGROUND: Probiotics are live microorganisms, generally either lactobacilli or bifidobacteria, which when administered in adequate amounts confer a health benefit to the host [1]. Due to the growing evidence of health benefits associated with their use, probiotics are of increasing interest and represent now a significant growth area in the functional foods industry [2]. However, to be effective, orally administered probiotics should survive preparation of dosage forms and passage through acidic environment of the gastrointestinal tract (GIT). Reaching the intestine, these microorganisms should be able to establish themselves, remain viable and perform their beneficial actions. In this context, oral formulations have to protect probiotic bacteria from gastric acidity and delay their release in the small intestine in order to allow their complete release in the colon.
\end{abstract}

OBJECTIVE: To evaluate effects of starch formulations of lactobacilli on their survival in gastric environment and probiotic properties.

METHODS: Nineteen Lactobacillus strains belonging to the species L. fermentum (14 strains), L. plantarum (4 strains), and L. rhamnosus (1 strain), were isolated from dairy products and probiotics, and were used in this study. Lactobacilli were cultured in de Man, Rogosa, Sharpe (MRS) broth (Merck, Germany) under microaerobic conditions at $37^{\circ} \mathrm{C}$.

Amylolytic activity of lactobacilli, cultured for 3-5 days on MRS agar supplemented with $1 \%$ soluble potato starch (SPS), was determined with iodine reagent (0.01 $\mathrm{M} \mathrm{I}_{2}-\mathrm{KI}$ solution).

Loading in starch was performed with L. plantarum 8PA3 bacteria ("Dry lactobacterin", Perm, Russia), which were resuspended to the concentration $10^{10}$ cells $/ \mathrm{mL}$ in $10 \mathrm{~mL}$ of $0.85 \% \mathrm{NaCl}$ solution and added to $90 \mathrm{~mL}$ of $2.5 \%$ SPS solution. Resulting mixture was frozen at $-18^{\circ} \mathrm{C}$ and then lyophilized (Martin Christ Alpha 1-2 LDplus, Germany).

Atomic force microscopy (AFM) images of formulated L. plantarum 8PA3 cells were acquired in air by a Solver P47H atomic force microscope (NT-MDT, Moscow, Russia).

Starch swelling and dissolution was studied in simulated colonic fluid (SCF), prepared according to [3] and in distilled water $(\mathrm{pH}=6.0)$ as control. Amylase from Aspergillus oryzae (A8220, Sigma) was added to the solutions to study the influence of amylase. The formulation form was examined visually during $14 \mathrm{~h}$ incubation time.

Fluorescence microscopy images were obtained with a Leica DM6000B (Germany) fluorescent microscope using Leica FW4000 software.

L. plantarum 8PA3 loaded in SPS were placed either in $\mathrm{HCl}$ solution (pH 2), or in $2 \%$ oxgall bile solution, or in $0.85 \% \mathrm{NaCl}$ solution. Viability was tested after 2,4 and $6 \mathrm{~h}$ incubation at $37^{\circ} \mathrm{C}$ by plating diluted aliquots onto MRS agar with subsequent counting of bacterial colony forming units 
(CFU). In addition, viability was determined using LIVE/DEAD BacLight bacterial viability kit L-7012 (Molecular Probes, Invitrogen) as described elsewhere [4]. Fluorescence in the stained samples was estimated with BD FACS Canto II (USA) flow cytometer or fluorescent microscope.

Nitric oxide (NO) production was assessed with DAF-FM DA and DAA fluorescent dyes as described earlier [4]. Each experiment was performed in triplicate.

RESULTS: In the present study we studied the probiotic composition comprising of SPS and bacteria L. plantarum 8PA3. We used AFM to confirm effective fixation of the cells to carbohydrate. The compositions were found to swell quickly $(\sim 5 \mathrm{~min})$ in aqueous solutions either containing amylase, or not. Tested starch formulations disintegrated during the first 5-10 min of incubation in amylase solutions whereas in amylase-free probes dissolution was less intensive (after $\sim 30 \mathrm{~min}$ ). Amylolysis of starch excipients was less pronounced in aqueous amylase solution than in SCF, supplemented with amylase. None of 19 studied Lactobacillus strains hydrolyzed SPS when growing on MRS agar supplemented with it. The amount of viable $L$. plantarum 8PA3 cells formulated with SPS was high and did not change when stored for 6 months at $4^{\circ} \mathrm{C}$. The bacterial viability tests also demonstrated that after $6 \mathrm{~h}$ treatment with $2 \%$ bile or $\mathrm{HCl}(\mathrm{pH} 2)$ L. plantarum $8 \mathrm{PA} 3$ exhibited increased sensitivity (viability $14 \%$ and $0.4 \%$, respectively). However, in similar conditions no significant differences were noticed between bacterial viability obtained for formulated with starch and non-formulated bacteria. Furthermore, we showed that loading into SPS had no effect on bacterial production of nitric oxide (NO) - a pluripotent regulatory molecule in human organism.

CONCLUSIONS: Overall, the results strongly support that formulation with polymeric matrices on the basis of SPS represent an appealing technology of probiotics production. It provides slow release of bacteria in target environment and does not alter their viability and NO biosynthesis. However, SPS excipient does not preserve the bacteria from harsh conditions of upper GIT. Therefore, we conclude that for oral administration the composition should be loaded in acid-resistant capsules.

Keywords: Probiotic, polymeric matrix, composition, soluble potato starch

Conflict of interest statement: The authors did not provide any information.

\section{References}

[1] FAO/WHO. Report on Joint FAO/WHO Expert Consultation on Evaluation of Health and Nutritional Properties of Probiotics in Food Including Powder Milk with Live Lactic Acid Bacteria. 2001 Oct. Available from: ftp://ftp.fao.org/es/ esn/food/probio_report_en.pdf

[2] Kanmani P, Satish Kumar R, Yuvaraj N, Paari KA, Pattukumar V, Arul V. Probiotics and its functionally valuable products - A review. Crit Rev Food Sci Nutr. 2013;53(6):641-58. doi: 10.1080/10408398.2011.553752

[3] Chen L, Li X, Pang Y, Lin L, Zhang X, Yu L. Resistant starch as a carrier for oral colon-targeting drug matrix system. J Mater Sci Mater Med. 2007 Nov;18(11):2199-203. Epub 2007 Aug 1. doi: 10.1007/s10856-007-3009-6

[4] Yarullina DR, Beuerlein K, Silkin NI, Ilinskaya ON. Nitric oxide production in probiotic Lactobacillus plantarum: Revision of the origin. JMRR. 2015;1(1):5-14. 